\title{
ALGEBRAICALLY INVARIANT EXTENSIONS OF $\sigma$-FINITE MEASURES ON EUCLIDEAN SPACE
}

\author{
KRZYSZTOF CIESIELSKI
}

\begin{abstract}
Let $G$ be a group of algebraic transformations of $\mathbf{R}^{n}$, i.e., the group of functions generated by bijections of $\mathbf{R}^{n}$ of the form $\left(f_{1}, \ldots, f_{n}\right)$ where each $f_{i}$ is a rational function with coefficients in $\mathbf{R}$ in $n$-variables. For a function $\gamma: G \rightarrow(0, \infty)$ we say that a measure $\mu$ on $\mathbf{R}^{n}$ is $\gamma$-invariant when $\mu(g[A])=\gamma(g) \cdot \mu(A)$ for every $g \in G$ and every $\mu$-measurable set $A$. We will examine the question: "Does there exist a proper $\gamma$-invariant extension of $\mu$ ?" We prove that if $\mu$ is $\sigma$-finite then such an extension exists whenever $G$ contains an uncountable subset of rational functions $H \subset\left(\mathbf{R}\left(X_{1}, \ldots, X_{n}\right)\right)^{n}$ such that $\mu\left(\left\{x: h_{1}(x)=h_{2}(x)\right\}\right)=0$ for all $h_{1}, h_{2} \in H, h_{1} \neq h_{2}$. In particular if $G$ is any uncountable subgroup of affine transformations of $\mathbf{R}^{n}, \gamma(g)$ is the absolute value of the Jacobian of $g \in G$ and $\mu$ is a $\gamma$-invariant extension of the $n$-dimensional Lebesgue measure then $\mu$ has a proper $\gamma$-invariant extension. The conclusion remains true for any $\sigma$-finite measure if $G$ is a transitive group of isometries of $\mathbf{R}^{n}$. An easy strengthening of this last corollary gives also an answer to a problem of Harazisvili.
\end{abstract}

\section{INTRODUCTION: NOTATION AND HISTORY}

Our terminology related to algebra, measure theory, set theory and model theory follows [ $\mathrm{La}, \mathrm{Ru}, \mathrm{Je}$ and $\mathrm{CK}$ ] respectively.

Throughout the paper a measure on a set $X$ will stand for a nontrivial positive $\sigma$-additive measure, i.e., a function $\mu: \mathscr{M} \rightarrow[0, \infty]$ defined on a $\sigma$-algebra $\mathscr{M}$ of subsets of $X$ containing all singletons such that

(i) $\mu\left(\bigcup_{i=0}^{\infty} A_{i}\right)=\sum_{i=0}^{\infty} \mu\left(A_{i}\right)$ for all pairwise disjoint sets $A_{i}$ from $\mathscr{M}$,

(ii) $\mu(\{x\})=0$ for all $x \in X$,

(iii) $0<\mu(A)<\infty$ for some $A \in \mathscr{M}$.

If $\mu: \mathscr{M} \rightarrow[0, \infty]$ is a measure on $X$ and $A \subset X$ then the inner measure of $A$ is defined in the standard way: $\mu_{*}(A)=\sup \{\mu(B): B \subset A \& B \in \mathscr{M}\}$.

A measure on $X$ is said to be $\sigma$-finite if $X$ is a countable union of sets of finite measure. A measure $\mu$ is complete if all subsets of every set of $\mu$ measure zero are $\mu$-measurable.

Received by the editors March 15, 1988 and, in revised form, June 22, 1988.

1980 Mathematics Subject Classification (1985 Revision). Primary 28C10; Secondary 14L35.

Key words and phrases. Invariant $\sigma$-finite measures, algebraic transformations of $\mathbf{R}^{n}$, isometries of $\mathbf{R}^{n}$.

The results of this paper have been presented at MAA and AMS Joint Mathematics Meeting, Phoenix, Arizona, January 1988 and at the Sixth Annual Auburn Miniconference on Real Analysis, Auburn, Alabama, April 1988. 
If $G$ is a group of bijections of a set $X$ then a measure $\mu$ on $X$ is said to be $G$-invariant provided $\mu$ is $\gamma$-invariant where $\gamma(g)=1$ for all $g \in G$.

For example, if $A_{n}$ is a group of affine transformations of $\mathbf{R}^{n}$ then every element of $A_{n}$ is uniquely represented as a superposition $T \circ L$ where $T$ is a translation and $L$ is a linear transformation of $\mathbf{R}^{n}$. Let $\gamma: A_{n} \rightarrow(0, \infty)$, where $\gamma(T \circ L)$ is defined as the absolute value of the Jacobian of $L$. Then $m$, the $n$-dimensional Lebesgue measure, is $\gamma$-invariant. Moreover, if $G_{n}$ is a group of isometries of $\mathbf{R}^{n}$ then $G_{n} \subset A_{n}$ and $m$ is $G_{n}$-invariant.

We say that a measure $\nu: \mathcal{N} \rightarrow[0, \infty]$ on a set $X$ is an extension of a measure $\mu: \mathscr{M} \rightarrow[0, \infty]$ defined on the same set $X$ if $\mathscr{M} \subset \mathscr{N}$ and $\nu(A)=$ $\mu(A)$ for every $A \in \mathscr{M}$. Moreover, an extension is proper if $\mathscr{M} \neq \mathscr{N}$.

For a group $G$ of bijections of a set $X$ we say that a set $N \subset X$ is $G$ absolutely negligible if for every $G$-invariant $\sigma$-finite measure $\mu$ on $X$ and for every countable set $\left\{g_{r}: r=0,1,2, \ldots\right\} \subset G$ we have $\mu_{*}\left(\bigcup_{r=0}^{\infty} g_{r}[N]\right)=0$ (or, equivalently, if for every $G$-invariant $\sigma$-finite measure $\mu$ on $X$ there exists a $G$-invariant extension $\nu$ of $\mu$ such that $\nu(N)=0$; compare Proposition $1.2(\mathrm{~b}))$.

We say that a bijection $g$ of $\mathbf{R}^{n}$ is an algebraic transformation of $\mathbf{R}^{n}$ if $g$ is generated by bijections of $\mathbf{R}^{n}$ from the set $\left(\mathbf{R}\left(X_{1}, \ldots, X_{n}\right)\right)^{n}$. For an algebraic transformation $g$ of $\mathbf{R}^{n}$ we say that $g$ is defined over the field $L \subset \mathbf{R}$ if $g$ is generated by some bijections of $\mathbf{R}^{n}$ from $\left(L\left(X_{1}, \ldots, X_{n}\right)\right)^{n}$. For example, the functions

$$
f(x, y)=\left(x^{3}+1,(y+7)^{5}\right), \quad g(x, y)=\left(x, y+\frac{1}{x^{2}+1}\right)
$$

and

$$
\left(f^{-1} \circ g\right)(x, y)=\left((x-1)^{1 / 3},\left(y+\frac{1}{x^{2}+1}\right)^{1 / 5}-7\right)
$$

are algebraic transformations of $\mathbf{R}^{2}$ defined over $\mathbf{Q}$. Notice also that isometries and, more generally, nonsingular affine transformations of $\mathbf{R}^{n}$ are algebraic transformations of $\mathbf{R}^{n}$ that belong to the set $\left(\mathbf{R}\left(X_{1}, \ldots, X_{n}\right)\right)^{n}$.

Now let $G$ be the group of all isometries of $\mathbf{R}^{n}$ and let $\mu$ be a $G$-invariant $\sigma$-finite measure on $\mathbf{R}^{n}$. Can we find a proper $G$-invariant extension of $\mu$ ?

This question has been discussed several times in the literature. In 1935 Szpilrajn proved that Lebesgue measure on $\mathbf{R}^{n}$ has a proper isometrically invariant extension (see [Sz]). In the same paper, he stated Sierpinski's question: "Does there exist a maximal isometrically invariant extension of Lebesgue measure on $\mathbf{R}^{n}$ ?" A negative answer to this question, i.e., the theorem "every isometrically invariant measure that extends Lebesgue measure on $\mathbf{R}^{n}$ has a proper isometrically invariant extension," was proved by several mathematicians. The first result of that kind was obtained independently by Pkhakadze (in 1958, see [Pk]) and Hulanicki (in 1962, see [Hu]) under the additional settheoretical assumption that there does not exist a real measurable cardinal less 
than or equal to continuum $2^{\omega}$, i.e., that there is no measure on $\mathbf{R}$ defined on all subsets of R. In 1977, Harazisvili got the full result stated above without any set-theoretical assumptions for the one dimensional case, i.e., for $n=1$ (see [Ha1]). Finally in 1983, Ciesielski and Pelc generalized Harazisvili's result to all $n$-dimensional Euclidean spaces $\mathbf{R}^{n}$ (see [CP]; for more historical details of this issue see also [Ci]). In the same paper Ciesielski and Pelc stated the problem of characterizing those groups $G$ of isometries of $\mathbf{R}^{n}$ for which every $\sigma$-finite $G$-invariant measure has a proper $G$-invariant extension (see [CP, p. 6]). A more technical version of the same problem, i.e., the problem of characterizing those groups $G$ of isometries of $\mathbf{R}^{n}$ for which $\mathbf{R}^{n}$ is a union of countable many $G$-absolutely negligible sets, was also stated by Harazisvili in [Ha2].

In the present paper we will consider a generalization of this problem to the case of $\gamma$-invariant measure where $\gamma: G \rightarrow(0, \infty)$ and $G$ is a group of algebraic transformations of $\mathbf{R}^{n}$. In particular our main theorem (see Abstract, or Theorem 3.1) implies that

"if $G$ is a transitive group of isometries of $\mathbf{R}^{n}$ then $\mathbf{R}^{n}$ is a countable union of $G$-absolutely negligible sets."

The above fact has been proved earlier by Harazisvili under the assumption of the continuum hypothesis (see [Ha2]). He also asked whether it is possible to remove this assumption from his theorem. Our results give an affirmative answer to this question.

The proof of our main theorem 3.1 uses a generalization of the technique of Ciesielski and Pelc [CP, Theorem 2.1, pp. 4-6]. The author wishes to thank Jan Mycielski for numerous important remarks about former versions of this paper. In particular it was Mycielski's suggestion to replace in the proof of [CP, Theorem 2.1] the linear basis of $\mathbf{R}$ over $\mathbf{Q}$ by a transcendence basis of $\mathbf{R}$ over $\mathbf{Q}$ and to study in this way algebraic transformations of $\mathbf{R}^{n}$. Compare also the paper of Weglorz [We, Theorem 2.4] which was influenced by Mycielski in a similar way.

The author wishes also to thank Piotr Zakrzewski for calling his attention to the paper of Harazisvili [Ha2] and for other helpful remarks.

\section{Measure theoretic PREliminaries}

In what follows we will need the following proposition essentially due to Szpilrajn (see [Sz, §2]).

Proposition 1.1. Let $\gamma: G \rightarrow(0, \infty)$ where $G$ is a group of bijections of a set $X$ and let $\mu: \mathscr{M} \rightarrow[0, \infty]$ be a $\gamma$-invariant measure on $X$. If a family $\mathscr{A}$ of subsets of $X$ is such that

(i) $\mathscr{A}$ is closed under countable union,

(ii) if $A \in \mathscr{A}$ and $g \in G$ then $g[A] \in \mathscr{A}$,

(iii) every $A \in \mathscr{A}$ has $\mu$ inner measure zero, 
then $\mu$ has a $\gamma$-invariant extension $\nu: \mathscr{N} \rightarrow[0, \infty]$ such that $\mathscr{A} \subset \mathscr{N}$ and $\nu(A)=0$ for every $A \in \mathscr{A}$.

The construction of such an extension is very simple. If $\mathscr{I}$ is an ideal of subsets of $X$ generated by the family $\mathscr{A}$, and $\mathscr{N}$ stands for a $\sigma$-algebra generated by $\mathscr{M} \cup \mathscr{I}$ then all elements of $\mathscr{N}$ are of the form $\left(M \cup I_{1}\right) \backslash I_{2}$ where $M \in \mathscr{M}$ and $I_{1}, I_{2} \in \mathscr{I}$. It is easy to see that $\nu: \mathscr{N} \rightarrow[0, \infty]$ such that $\nu\left(\left(M \cup I_{1}\right) \backslash I_{2}\right)=\mu(M)$ is a well-defined $\gamma$-invariant measure on $X$ extending $\mu$.

In the proof of the next proposition, we use a method which goes back to Harazisvili's paper [Ha1] (see also [CP, Proposition 1.9, p. 4]).

Proposition 1.2. Let $G$ be a group of bijections of $X, \gamma: G \rightarrow(0, \infty)$ and let $\mu$ be a $\gamma$-invariant $\sigma$-finite measure on $X$.

(a) If $N \subset X$ is such that there is an uncountable set $H \subset G$ such that $\mu_{*}\left(h_{1}[N] \cap h_{2}[N]\right)=0$, for distinct $h_{1}, h_{2} \in H$, then $\mu_{*}(N)=0$.

(b) If $N \subset X$ is such that for every countable set $\left\{g_{r}: r=0,1,2, \ldots\right\} \subset G$ we have $\mu_{*}\left(\bigcup_{r=0}^{\infty} g_{r}[N]\right)=0$ then there exists a $\gamma$-invariant extension $\nu$ of $\mu$ such that $\nu(N)=0$.

(c) Moreover if $X=\bigcup_{k=0}^{\infty} N_{k}$ where each $N_{k}$ satisfies the assumption of (b) then $\mu$ has a proper $\gamma$-invariant extension.

Proof. (a) If $M \in \mathscr{M}$ is a subset of $N$ then $\mu\left(h_{1}[M] \cap h_{2}[M]\right)=0$ for every distinct $h_{1}, h_{2}$ from $H$. But $\mu(h[M])=\gamma(h) \cdot \mu(M)$ and $\gamma(h) \neq 0$ for every $h$ from $H$. Hence, $\sigma$-finiteness of $\mu$ implies that $\mu(M)=0$ and so $\mu_{*}(N)=0$.

(b) By Proposition 1.1 it is enough to notice that every element of the family $\mathscr{A}=\left\{\bigcup_{r=0}^{\infty} g_{r}[N]: g_{r} \in G\right.$ for $\left.r=0,1,2, \ldots\right\}$ has $\mu$ inner measure 0 .

(c) By part (b), for each $k=0,1,2, \ldots$ there is a $\gamma$-invariant extension $\nu_{k}$ of $\mu$ such that $\nu_{k}\left(N_{k}\right)=0$. But all $N_{k}$ 's cannot have $\mu$ measure zero. So some $\nu_{k}$ must be a proper extension of $\mu$.

In what follows, we will also use the following well-known fact. For the complex case the proof (using the Jensen's Inequality) can be found in [GR, p. 9]. The direct proof follows also from Fubini's theorem.

Proposition 1.3. If $f: \mathbf{R}^{n} \rightarrow \mathbf{R}$ is a nonzero real analytic function then the set $Z=\left\{a \in \mathbf{R}^{n}: f(a)=0\right\}$ has Lebesgue measure zero. In particular, if $h, g \in$ $\left(\mathbf{R}\left(X_{1}, \ldots, X_{n}\right)\right)^{n}$ are different algebraic transformations of $\mathbf{R}^{n}$ then the set $\left\{a \in \mathbf{R}^{n}: h(a)=g(a)\right\}$ has Lebesgue measure zero.

\section{Algebraic PRELIMINARIES}

A field $L \subset \mathbf{R}$ is said to be algebraically closed in $\mathbf{R}$ if $L=M \cap \mathbf{R}$ where $M \subset \mathbf{C}$ is an algebraic closure of $L$. Notice, that an algebraically closed field in $\mathbf{R}$ is real closed (i.e. satisfies the theory of real closed fields) in the sense defined in [CK or Ro]. The smallest field algebraically closed in $\mathbf{R}$ containing $L \subset \mathbf{R}$ is called a real closure of $L$ and it will be denoted by $\mathrm{cl}_{\mathbf{R}}(L)$. The algebraic closure of a field $K$ will be denoted by $\operatorname{cl}(K)$. 
The next proposition will be used only in the case of algebraic transformation $g$ such that $g^{-1} \in\left(\mathbf{R}\left(X_{1}, \ldots, X_{n}\right)\right)^{n}$. In this case this is a well-known fact and can be proved using standard algebraic technic. However we like to prove it in more general form (that possibly can be used to answer Problem 3 stated in the end of the paper). For this we will need the following model-theoretic definition (compare e.g. [CK]).

A model $\mathscr{L}$ is said to be an elementary submodel of a model $\mathscr{R}$ if $\mathscr{L} \subset \mathscr{R}$ and for every first order formula $\varphi\left(x_{1}, \ldots, x_{m}\right)$ and any parameters $a_{1}, \ldots, a_{m}$ from $\mathscr{L}$ the model $\mathscr{L}$ satisfies $\varphi\left(a_{1}, \ldots, a_{m}\right)$ if and only if $\mathscr{R}$ satisfies $\varphi\left(a_{1}, \ldots, a_{m}\right)$.

A theory $T$ is said to be model complete if and only if for all models $\mathscr{L}$ and $\mathscr{R}$ of $T$, if $\mathscr{L} \subset \mathscr{R}$ then $\mathscr{L}$ is an elementary submodel of $\mathscr{R}$.

We need the following important theorem of A. Robinson (see [CK, p. 110] or $[$ Ro, $\S 3.3])$.

Theorem 2.1. The theory $T$ of real closed fields is model complete. In particular if $L \subset \mathbf{R}$ is a real closed field then $L$ is an elementary submodel of $\mathbf{R}$.

As a corollary of this fact we easily obtain

Proposition 2.1. If $g$ is an algebraic transformation of $\mathbf{R}^{n}$ defined over a real closed field $L \subset \mathbf{R}$ then

$$
g\left[L^{n}\right]=L^{n} .
$$

Proof. A first order formula $\varphi\left(x_{1}, \ldots, x_{n}, y_{1}, \ldots, y_{n}\right)$ defined by $g\left(x_{1}, \ldots, x_{n}\right)$ $=\left(y_{1}, \ldots, y_{n}\right)$ has as its parameters only elements from $L$. If $a=$ $\left(a_{1}, \ldots, a_{n}\right) \in L^{n}$ then $\mathbf{R}$ satisfies $\exists y_{1} \ldots \exists y_{n} \varphi\left(a_{1}, \ldots, a_{n}, y_{1}, \ldots, y_{n}\right)$ and so does $L$ (by Theorem 2.1), i.e. $g\left(a_{1}, \ldots, a_{n}\right) \in L^{n}$. This proves $g\left[L^{n}\right] \subset$ $L^{n}$. To show the converse inclusion it is enough to consider the formula $\exists x_{1} \ldots \exists x_{n} \varphi\left(x_{1}, \ldots, x_{n}, a_{1}, \ldots, a_{n}\right)$.

\section{THE MAIN THEOREM}

From now on let $\mathscr{B}$ denote a transcendence base of $\mathbf{R}$ over $\mathbf{Q}$.

Now we are ready to prove our main lemma.

Lemma 3.1. Let $H \subset\left(\mathbf{R}\left(X_{1}, \ldots, X_{n}\right)\right)^{n}$ be an uncountable set of algebraic transformations of $\mathbf{R}^{n}$. Then there exists an uncountable set $H^{\prime} \subset H$, a finite set $A \subset \mathscr{B}$ and, for every $h \in H^{\prime}$, a finite set $A_{h} \subset \mathscr{B} \backslash A$ with the following properties:

(1) each $h \in H^{\prime}$ (and so $\left.h^{-1}\right)$ is defined over the field $\operatorname{cl}_{\mathbf{R}}\left(\mathbf{Q}\left(A \cup A_{h}\right)\right)$;

(2) $A_{h_{1}} \cap A_{h_{2}}=\varnothing$ for distinct $h_{1}, h_{2} \in H^{\prime}$;

(3) for every $h_{1}, h_{2} \in H^{\prime}$ if $L=\operatorname{cl}_{\mathbf{R}}\left(\mathbf{Q}\left(\mathscr{B} \backslash\left(A_{h_{1}} \cup A_{h_{2}}\right)\right)\right)$ then $a \in h_{1}^{-1}\left[L^{n}\right] \cap$ $h_{2}^{-1}\left[L^{n}\right]$ implies $h_{1}(a)=h_{2}(a)$, i.e., $h_{1}^{-1}\left[L^{n}\right] \cap h_{2}^{-1}\left[L^{n}\right] \subset\left\{a: h_{1}(a)=h_{2}(a)\right\}$. 
Proof. In the definition of each $h \in H$ we use only finitely many parameters (i.e. coefficients) so for every $h \in H$ there exists a finite set $B_{h} \subset \mathscr{B}$ such that

$$
h=\left(h_{1}, \ldots, h_{n}\right) \in\left[\mathrm{cl}_{\mathbf{R}}\left(\mathbf{Q}\left(B_{h}\right)\right)\left(X_{1}, \ldots, X_{n}\right)\right]^{n} .
$$

Using for the family $\left\{B_{h}: h \in H\right\}$ the $\Delta$-system argument (see e.g. [Je, Lemma 22.6, p. 226]) we can find an uncountable set $H_{0} \subset H$, a finite set $A \subset \mathscr{B}$, a natural number $m$ and, for every $h \in H_{0}$, a set $A_{h}$ such that

(i) $B_{h}=A \cup A_{h}$, and $A \cap A_{h}=\varnothing$,

(ii) $A_{h_{1}} \cap A_{h_{2}}=\varnothing$ for distinct $h_{1}, h_{2} \in H_{0}$,

(iii) $A_{h}$ has exactly $m$ elements.

Thus for the family $H_{0}$, the sets $A, A_{h} \quad\left(h \in H_{0}\right)$ already satisfy (1) and (2). Therefore it is enough to find an uncountable $H^{\prime} \subset H_{0}$ which satisfies (3). We will do this in such a way that all elements of $H^{\prime}$ will have the same definitions with parameters from $\mathscr{B}$.

Let $Z=\left\{Z_{1}, \ldots, Z_{m}\right\}$ be a set of variables and, for $h \in H_{0}$, let $\sigma_{h}^{\prime}: A_{h} \rightarrow Z$ be a bijection. Then we can extend $\sigma_{h}^{\prime}$ to a field isomorphism $\sigma_{h}^{\prime \prime}$ from $\operatorname{cl}(\mathbf{Q}(\mathscr{B}))=\mathbf{C}$ to $\operatorname{cl}\left(\mathbf{Q}\left(\mathscr{B} \backslash A_{h}\right)(Z)\right)$ in such a way that $\sigma_{h}^{\prime \prime}(a)=a$ for every $a \in \operatorname{cl}\left(\mathbf{Q}\left(\mathscr{B} \backslash A_{h}\right)\right)$. Let us extend $\sigma_{h}^{\prime \prime}$ to $\sigma_{h}:\left[\operatorname{cl}(\mathbf{Q}(\mathscr{B}))\left(X_{1}, \ldots, X_{n}\right)\right]^{n} \rightarrow$ $\left[\operatorname{cl}\left(\mathbf{Q}\left(\mathscr{B} \backslash A_{h}\right)(Z)\right)\left(X_{1}, \ldots, X_{n}\right)\right]^{n}$. But $\sigma_{h}(h) \in\left[\operatorname{cl}(\mathbf{Q}(A \cup Z))\left(X_{1}, \ldots, X_{n}\right)\right]^{n}$ and the field $\operatorname{cl}(\mathbf{Q}(A \cup Z))$ is countable.

Define $H^{\prime} \subset H_{0}$ as an uncountable set with the property

$$
\sigma_{h_{1}}\left(h_{1}\right)=\sigma_{h_{2}}\left(h_{2}\right) \text { for every } h_{1}, h_{2} \in H^{\prime} .
$$

We prove that $H^{\prime}$ satisfies (3).

Let $a \in h_{1}^{-1}\left[L^{n}\right] \cap h_{2}^{-1}\left[L^{n}\right]$, where $L=\operatorname{cl}_{\mathbf{R}}\left(\mathbf{Q}\left(\mathscr{B} \backslash\left(A_{h_{1}} \cup A_{h_{2}}\right)\right)\right)$ and $h_{1}, h_{2} \in$ $H^{\prime}$. Notice that $a \in L^{n}$ as, by Proposition 2.1, (1) and (2),

$$
\begin{aligned}
a \in & h_{1}^{-1}\left[L^{n}\right] \cap h_{2}^{-1}\left[L^{n}\right] \subset h_{1}^{-1}\left[\left(\operatorname{cl}_{\mathbf{R}}\left(\mathbf{Q}\left(\mathscr{B} \backslash A_{h_{2}}\right)\right)\right)^{n}\right] \cap h_{2}^{-1}\left[\left(\mathrm{cl}_{\mathbf{R}}\left(\mathbf{Q}\left(\mathscr{B} \backslash A_{h_{1}}\right)\right)\right)^{n}\right] \\
& =\left(\mathrm{cl}_{\mathbf{R}}\left(\mathbf{Q}\left(\mathscr{B} \backslash A_{h_{2}}\right)\right)^{n} \cap\left(\mathrm{cl}_{\mathbf{R}}\left(\mathbf{Q}\left(\mathscr{B} \backslash A_{h_{1}}\right)\right)\right)^{n}=L^{n} .\right.
\end{aligned}
$$

Put $h_{1}(a)=b_{1}$ and $h_{2}(a)=b_{2}$. Thus $b_{1}, b_{2} \in L^{n}$. We have to prove that $b_{1}=b_{2}$. But, by $(*)$ and the fact that $\sigma_{h_{1}}(c)=c=\sigma_{h_{2}}(c)$ for every $c \in L^{n}$,

$$
\begin{aligned}
b_{1} & =\sigma_{h_{1}}\left(b_{1}\right)=\sigma_{h_{1}}\left(h_{1}(a)\right)=\sigma_{h_{1}}\left(h_{1}\right)\left(\sigma_{h_{1}}(a)\right)=\sigma_{h_{1}}\left(h_{1}\right)(a) \\
& =\sigma_{h_{2}}\left(h_{2}\right)(a)=\sigma_{h_{2}}\left(h_{2}\right)\left(\sigma_{h_{2}}(a)\right)=\sigma_{h_{2}}\left(h_{2}(a)\right)=\sigma_{h_{2}}\left(b_{2}\right)=b_{2} .
\end{aligned}
$$

This finishes the proof of Lemma 3.1.

As a next step we will prove an essential part of the assumptions of Proposition 1.2.

Lemma 3.2. If $G$ is a group of algebraic transformations of $\mathbf{R}^{n}$ and $H \subset$ $\left(\mathbf{R}\left(X_{1}, \ldots, X_{n}\right)\right)^{n}$ is an uncountable subset of $G$ then there exists a countable 
family of sets $\left\{N_{k}: k=0,1,2, \ldots\right\}$ such that $\mathbf{R}^{n}=\bigcup_{k=0}^{\infty} N_{k}$ and that each $N_{k}$ satisfies the condition:

for every countable set $\left\{g_{r}: r=0,1,2, \ldots\right\} \subset G$ there is an uncountable set $H_{0} \subset H$ such that for every distinct $h_{1}, h_{2} \in H_{0}$

$$
h_{1}^{-1}\left[\bigcup_{r=0}^{\infty} g_{r}\left[N_{k}\right]\right] \cap h_{2}^{-1}\left[\bigcup_{r=0}^{\infty} g_{r}\left[N_{k}\right]\right] \subset\left\{a \in \mathbf{R}^{n}: h_{1}(a)=h_{2}(a)\right\} \text {. }
$$

Proof. Let $\mathscr{B}$ be a transcendence base of $\mathbf{R}$ over $Q$ and let $H^{\prime} \subset H, A$ and $A_{h}$ be as in Lemma 3.1. We choose an increasing sequence $\mathscr{B}_{0} \subset \mathscr{B}_{1} \subset \mathscr{B}_{2} \subset \ldots$ of subsets of $\mathscr{B}$ in such a way that $\mathscr{B}=\bigcup_{k=1}^{\infty} \mathscr{B}_{k}$ and for every $k$ the set

$$
H^{k}=\left\{h \in H^{\prime}: A_{h} \subset \mathscr{B}_{k+1} \backslash \mathscr{B}_{k}\right\}
$$

is uncountable.

Define $N_{k}=\left[\mathrm{cl}_{\mathbf{R}}\left(\mathbf{Q}\left(\mathscr{B}_{k}\right)\right)\right]^{n}$. Then $\bigcup_{k=0}^{\infty} N_{k}=\mathbf{R}^{n}$.

Let us fix $\left\{g_{r}: r=0,1,2, \ldots\right\} \subset G$ and a natural number $k$. Choose also a countable set $\mathscr{A} \subset \mathscr{B}$ such that $A \subset \mathscr{A}$ and every $g_{r}$ is defined over $\mathrm{cl}_{\mathbf{R}}(\mathbf{Q}(\mathscr{A}))$. Let $H_{0}=\left\{h \in H^{k+1}: A_{h} \cap \mathscr{A}=\varnothing\right\}$.

By $(*)$ the set $H_{0}$ is uncountable.

Let us fix arbitrary distinct $h_{1}, h_{2} \in H_{0}$ and let $L=\operatorname{cl}_{\mathbf{R}}\left(\mathbf{Q}\left(\mathscr{B} \backslash\left(A_{h_{1}} \cup A_{h_{2}}\right)\right)\right)$. Then, by $(*)$ and definitions of $H_{0}$ and $N_{k}$, we can conclude that $N_{k} \subset L^{n}$ and the $g_{r}$ 's are defined over $L$. Hence, by Proposition 2.1,

$$
\begin{aligned}
h_{1}^{-1}\left[\bigcup_{r=0}^{\infty} g_{r}\left[N_{k}\right]\right] & \cap h_{2}^{-1}\left[\bigcup_{r=0}^{\infty} g_{r}\left[N_{k}\right]\right] \subset h_{1}^{-1}\left[\bigcup_{r=0}^{\infty} g_{r}\left[L^{n}\right]\right] \\
& \cap h_{2}^{-1}\left[\bigcup_{r=0}^{\infty} g_{r}\left[L^{n}\right]\right]=h_{1}^{-1}\left[L^{n}\right] \cap h_{2}^{-1}\left[L^{n}\right]
\end{aligned}
$$

and, by (3) of Lemma 3.1, $h_{1}^{-1}\left[L^{n}\right] \cap h_{2}^{-1}\left[L^{n}\right] \subset\left\{a: h_{1}(a)=h_{2}(a)\right\}$.

Therefore

$$
h_{1}^{-1}\left[\bigcup_{r=0}^{\infty} g_{r}\left[N_{k}\right]\right] \cap h_{2}^{-1}\left[\bigcup_{r=0}^{\infty} g_{r}\left[N_{k}\right]\right] \subset h_{1}^{-1}\left[L^{n}\right] \cap h_{2}^{-1}\left[L^{n}\right] \subset\left\{a: h_{1}(a)=h_{2}(a)\right\} \text {. }
$$

This finishes the proof of Lemma 3.2.

Theorem 3.1. Let $G$ be a group of algebraic transformations of $\mathbf{R}^{n}, \gamma: G \rightarrow$ $(0, \infty)$ and let $\mu$ be a $\gamma$-invariant $\sigma$-finite measure on $\mathbf{R}^{n}$. If $G$ has an uncountable subset $H \subset\left(\mathbf{R}\left(X_{1}, \ldots, X_{n}\right)\right)^{n}$ with the property

$$
\mu_{*}\left(\left\{a: h_{1}(a)=h_{2}(a)\right\}\right)=0 \text { for every } h_{1}, h_{2} \in H, h_{1} \neq h_{2}
$$

then $\mu$ has a proper $\gamma$-invariant extension.

Proof. By (3.2) and Lemma 3.2 we have $\mathbf{R}^{n}=\bigcup_{k=0}^{\infty} N_{k}$ where, by Proposition $1.2(\mathbf{a}), \mu_{*}\left(\bigcup_{r=0}^{\infty} g_{r}\left[N_{k}\right]\right)=0$ for every countable set $\left\{g_{r}: r=0,1,2, \ldots\right\} \subset G$ 
and every $k=0,1,2, \ldots$ Hence, by Proposition 1.2(c), $\mu$ has a proper $\gamma$-invariant extension.

Corollary 3.1. Let $G$ be a group of algebraic transformations of $\mathbf{R}^{n}, \gamma: G \rightarrow$ $(0, \infty)$ and let $\mu$ be a $\gamma$-invariant $\sigma$-finite measure on $\mathbf{R}^{n}$. If at least one of the following conditions holds

(C1) $G$ contains uncountably many translations;

(C2) $\mu$ extends the $n$-dimensional Lebesgue measure and the set $G \cap$ $\left(\mathbf{R}\left(X_{1}, \ldots, X_{n}\right)\right)^{n}$ is uncountable;

then $\mu$ has a proper $\gamma$-invariant extension.

Proof. It is enough to show that both (C1) and (C2) imply (3.2).

If (C1) holds and $H$ is an uncountable set of translations then for every $h_{1}, h_{2} \in H, h_{1} \neq h_{2}$ the set $\left\{a: h_{1}(a)=h_{2}(a)\right\}$ is empty, so (3.2) is satisfied.

If (C2) holds then (3.2) is implied by Proposition 1.3.

To solve Harazisvili's problem we will need the following lemma due to Harazisvili (see [Ha2, Remark 2, p. 507]).

Lemma 3.3. Let $G$ be a transitive group of isometries of $\mathbf{R}^{n}$, i.e., such that for every $a, b \in \mathbf{R}^{n}$ there exists $g \in G$ with the property $g(a)=b$. If $A \subset \mathbf{R}^{n}$ is a countable union of proper affine hyperplanes of $\mathbf{R}^{n}$ than $A$ is $G$-absolutely negligible.

Proof. For $k \leq n$ let $\mathscr{A}_{k}$ denote the family of countable unions of affine hyperplanes of $\mathbf{R}^{n}$ of dimension less than $k$. We prove by induction on $k \leq n$ that elements of $\mathscr{A}_{k}$ are $G$-absolutely negligible.

So let $k<n$ be such that the elements of $\mathscr{A}_{k}$ are $G$-absolutely negligible.

Let us fix an arbitrary $A \in \mathscr{A}_{k+1}$, a $G$-invariant $\sigma$-finite measure $\mu$ on $\mathbf{R}^{n}$ and a countable set $\left\{g_{r}: r=0,1,2, \ldots\right\} \subset G$. By Proposition 1.2(a) it is enough to find a sequence $\left\{h_{\zeta}: \zeta<\omega_{1}\right\} \subset G$ such that for every $\zeta<\eta<\omega_{1}$

$$
\mu_{*}\left(h_{\zeta}\left[\bigcup_{r=0}^{\infty} g_{r}[A]\right] \cap h_{\eta}\left[\bigcup_{r=0}^{\infty} g_{r}[A]\right]\right)=0 .
$$

We will construct it by transfinite induction.

So let us assume that for some $\xi<\omega_{1}$ we have already constructed $\left\{h_{\zeta}: \zeta<\right.$ $\xi\} \subset G$ such that the condition (a) is satisfied for every $\zeta<\eta<\xi$. Let $A_{i}$ and $H_{j}(i, j=0,1,2, \ldots)$ be affine hyperplanes of $\mathbf{R}^{n}$ of dimensions less than or equal to $k$ and such that

$$
\bigcup_{r=0}^{\infty} g_{r}[A]=\bigcup_{i=0}^{\infty} A_{i} \text { and } \bigcup_{\zeta<\xi} h_{\zeta}\left[\bigcup_{r=0}^{\infty} g_{r}[A]\right]=\bigcup_{j=0}^{\infty} H_{j} .
$$

We have to find $h_{\xi}$ such that

$$
\mu_{*}\left(h_{\xi}\left[\bigcup_{i=0}^{\infty} A_{i}\right] \cap \bigcup_{j=0}^{\infty} H_{j}\right)=0 .
$$


But if $h_{\xi}\left[A_{i}\right] \neq H_{j}$ then $h_{\xi}\left[A_{i}\right] \cap H_{j} \in \mathscr{A}_{k}$, i.e., by inductive hypothesis, it is enough to construct $h_{\xi} \in G$ such that

$$
h_{\xi}\left[A_{i}\right] \neq H_{j} \quad \text { for every } i, j=0,1,2, \ldots \text {. }
$$

Let $w \in \mathbf{R}^{n}$ represents a vector in $\mathbf{R}^{n}$ such that $w$ is not parallel to any $H_{j}$ $(j=0,1,2, \ldots)$. Then for different reals $a, b$ the distances

$$
\operatorname{dist}\left(0, a \cdot w+H_{j}\right) \neq \operatorname{dist}\left(0, b \cdot w+H_{j}\right) \quad \text { for every } j=0,1,2, \ldots
$$

So we can choose $b \in \mathbf{R}$ such that

(c) $\quad \operatorname{dist}\left(0,-b \cdot w+H_{j}\right) \neq \operatorname{dist}\left(0, A_{i}\right)$ for every $i, j=0,1,2, \ldots$ (b).

Now let $h_{\xi} \in G$ be such that $h_{\xi}(0)=b \cdot w$. We prove that such $h_{\xi}$ satisfies

By way of contradiction let us assume that for some $i$ and $j$

$$
h_{\xi}\left[A_{i}\right]=H_{j} \text {. }
$$

But $h_{\xi}=T \circ L$, where $L$ is an isometry of $\mathbf{R}^{n}$ preserving origin and $T$ is a translation such that $T(x)=x+b \cdot w$ for every $x \in \mathbf{R}^{n}$. Hence, by (d), $L\left[A_{i}\right]=T^{-1}\left[H_{j}\right]=-b \cdot w+H_{j}$ and so

$$
\operatorname{dist}\left(0,-b \cdot w+H_{j}\right)=\operatorname{dist}\left(0, L\left[A_{i}\right]\right)=\operatorname{dist}\left(0, A_{i}\right)
$$

contradicting (c).

Thus we proved that $h_{\xi}$ satisfies (b). This finishes the proof of the lemma.

Theorem 3.2. If $G$ is a transitive group of isometries of $\mathbf{R}^{n}$ then $\mathbf{R}^{n}$ is a countable union of $G$-absolutely negligible sets. In particular every $\sigma$-finite $G$ invariant measure on $\mathbf{R}^{n}$ has a proper G-invariant extension.

Proof. Let $\left\{N_{k}: k=0,1,2, \ldots\right\}$ be the family given in Lemma 3.2 where $H=$ $G$. Then by Lemma 3.3 and Proposition $1.2\left(\right.$ a) we have $\mu_{*}\left(\bigcup_{r=0}^{\infty} g_{r}[N]\right)=0$ for every countable set $\left\{g_{r}: r=0,1,2, \ldots\right\} \subset G$ and every $k=0,1,2, \ldots$. Hence each $N_{k}$ is $G$-absolutely negligible.

\section{GENERALIZATIONS, EXAMPLES AND PROBLEMS}

1. Let us remark first that although we have stated Theorem 3.1 only for measures on $\mathbf{R}^{n}$ the theorem can be generalized for measures on $K^{n}$ where $K$ is either a real closed or algebraically closed field, since the theory of algebraic closed fields is also model complete (see [CK, p. 110]). Moreover, in the case of algebraically closed fields, the assumptions that $H \subset\left(K\left(X_{1}, \ldots, X_{n}\right)\right)^{n}$ may be dropped.

2. If $X \subset K^{n}$ where $K$ is as above and we define algebraic transformations on $X$ in natural way, i.e., by functions generated by bijections of $X$ from $\left(K\left(X_{1}, \ldots, X_{n}\right)\right)^{n}$, then we can prove Theorem 3.1 for measures on $X$. In particular we can conclude that it does not exist a maximal isometrically invariant extension of Lebesgue measure on $n$-dimensional sphere $S^{n}$. 
3. Theorem 3.1 and its generalizations as in 1 and 2 can be also proved for complex measures (see [Ru, Chapter 6]).

4. For the cardinal number $\kappa$ we say that a measure $\mu$ on a set $X$ is $\kappa$-finite if $X$ is a union of $\kappa$ many sets of finite measure. Theorem 3.1 can be also generalized in the following way:

"Let $\kappa$ be a cardinal number, $G$ be a group of algebraic transformations of $\mathbf{R}^{n}, \gamma: G \rightarrow(0, \infty)$ and let $\mu$ be a $\gamma$-invariant $\kappa$-finite measure on $\mathbf{R}^{n}$. If $G$ has a subset $H \subset\left(\mathbf{R}\left(X_{1}, \ldots, X_{n}\right)\right)^{n}$ of power greater than $\kappa$ with the property

$$
\left\{a: h_{1}(a)=h_{2}(a)\right\}=\varnothing \text { for every } h_{1}, h_{2} \in H, h_{1} \neq h_{2},
$$

then $\mu$ has a proper $\gamma$-invariant extension."

5. In 4 condition $(*)$ can be replaced by the original condition (3.2) if we assume in addition that the measure $\mu$ is $\kappa^{+}$-additive.

6. We can also generalize the results from 4 and 5 in the way described in 1 and 2 .

7. By 4 , if in particular $\kappa$ is less than continuum $2^{\omega}, G$ is a group of all isometries of $\mathbf{R}^{n}$ and $\mu$ is a $\kappa$-finite $G$-invariant measure then there exists a proper $G$-invariant extension of $\mu$. However for $\kappa$ equal to continuum $2^{\omega}$ this cannot be proved as it was shown in [CP, Theorem 3.1].

8. An interesting example, suggested to the author by Jan Mycielski, can be obtained by considering a hyperbolic space $H^{n}$ for $n \geq 2$. If we identify $H^{n}$ with the model $\left\{\left(a_{1}, \ldots, a_{n+1}\right) \in \mathbf{R}^{n+1}: a_{n+1}>0\right\}$ then the group $G$ of all isometries of $H^{n}$ is a group of algebraic transformations of $\mathbf{R}^{n}$ and contains uncountably many translations. Moreover $G$ is not a subgroup of a group of affine transformations of $\mathbf{R}^{n}$ (see [MW or Be]). Let $\nu$ be the hyperbolic invariant measure on $H^{n}$ induced by the Haar measure on $G$. So $\nu$ is a $G$ invariant $\sigma$-finite measure on $H^{n}$. Using the previous remarks and Corollary 3.1 we may conclude that the measure $\nu$ does not have a maximal $G$-invariant extension.

9. Now we discuss the assumptions of Theorem 3.1, in particular condition (3.2).

First we prove that uncountability of $H \subset G$ is essential (compare [Pe, Proposition 2.3, p. 14]).

Let $G_{0}$ be a group of all translations of $\mathbf{R}^{1}$ by rational numbers and let $V$ be a Vitali set, i.e., $V \cap H$ is a one element set for each orbit $H$ of $G_{0}$. If we assume that there is a real measurable cardinal less than or equal to continuum (see [Je]) then there is a measure $\nu_{0}: \mathscr{P}(V) \rightarrow[0,1]$, where $\mathscr{P}(V)$ is a family of all subsets of the set $V$. Define a measure $\mu: \mathscr{P}\left(\mathbf{R}^{1}\right) \rightarrow[0, \infty]$ by

$$
\mu(A)=\sum_{g \in G_{0}} \nu_{0}\left(g^{-1}[g[V] \cap A]\right) .
$$

It is easy to see that $\mu$ is $G_{0}$-invariant and $\sigma$-finite. But $\mu$ is defined on all subsets of $\mathbf{R}^{1}$ so it cannot have any proper extension. 
10. It can be also proved that if there is a real measurable cardinal less than or equal to the continuum then for every countable group $G$ of bijections of $\mathbf{R}^{1}$ there exists a $G$-invariant measure defined on $\mathscr{P}\left(\mathbf{R}^{1}\right)$, however this needs a little more careful definition.

11. The group $G_{0}$ defined in 9 is related to an interesting open problem of Andrzej Pelc (see [Pe, p. 27]).

Problem 1. Let $\mu$ be a $G_{0}$-invariant extension of Lebesgue measure on $\mathbf{R}^{1}$. Does there exist a proper $G_{0}$-invariant extension of $\mu$ ?

12. The next example shows that we have to assume about $G$ something more than only uncountability.

Example. Let $G^{\prime}$ be the group of all rotations of $\mathbf{R}^{2}$ about the origin and let $\nu: \mathscr{P}\left(\mathbf{R}^{2}\right) \rightarrow[0, \infty]$ be such that $\nu(A)=1$ when $(0,0) \in A$ and $\nu(A)=0$ otherwise. $\nu$ does not vanish at points, but still it is a $G^{\prime}$-invariant measure. To correct this let $\mu$ and $G_{0}$ be as in Example 2 and let $\mu_{1}: \mathscr{P}\left(\mathbf{R}^{3}\right) \rightarrow[0, \infty]$ be a product measure of $\nu$ and $\mu$, i.e., $\mu_{1}(A)=\mu(\{x:(0,0, x) \in A\})$. Then $\mu$ is $\sigma$-finite and $G_{1}$-invariant, where the group $G_{1}=\left\{\left(g^{\prime}, g^{\prime \prime}\right): g^{\prime} \in G^{\prime}\right.$ and $\left.g^{\prime \prime} \in G_{1}\right\}$ is uncountable. It is also obvious that $\mu_{1}$ does not have any proper extension.

13. The reason that this example works is that $\mu_{1}$ is concentrated on a set $S=\{0\} \times\{0\} \times \mathbf{R}$ while $g[S]=S$ for every $g \in G_{1}$ and the group $\left\{\left.g\right|_{S}: g \in G_{1}\right\}$ is countable. This suggests the following

Definition. Let $G$ be a group of bijections of a set $X$ and $\mu$ be a $G$-invariant measure on $X$. We say that $G$ is $\mu$-essentially countable if there is a set $S \subset X$ such that $\mu(X \backslash S)=0, g[S]=S$ for all $g \in G$ and the group $\left\{\left.g\right|_{S}: g \in G\right\}$ is countable.

Problem 2. Let $G$ be a group of algebraic transformations of $\mathbf{R}^{n}$ and $\mu$ be a $G$-invariant $\sigma$-finite measure of $\mathbf{R}^{n}$ such that $G$ is not $\mu$-essentially countable. Does $\mu$ have a proper $G$-invariant extension?

Recently the author has been informed that Piotr Zakrzewski proved the following result connected with the Problem 2: "If $G$ is a group of isometries of $\mathbf{R}^{n}$ and $\mu: \mathscr{P}\left(\mathbf{R}^{n}\right) \rightarrow[0, \infty]$ is $G$-invariant then the group $G$ is $\mu$-essentially countable."

14. In the next example we will construct a $\gamma$-invariant measure $\mu$ on $\mathbf{R}^{1}$ where $\gamma$ will not be given in a classical way by Jacobian.

Example. Let $G_{0}=\left\{x^{3^{n}}: n \in \mathbf{Z}\right\}$ be a group of transformations of $\mathbf{R}^{1}$ and let $V \subset \mathbf{R}^{1} \backslash\{0\}$ be such that $(V \cup\{0\}) \cap H$ contains exactly one element for every orbit $H$ of $G$. Let $\mu_{0}: \mathscr{P}(V) \rightarrow[0,1]$ be a measure. For $n \in \mathbf{Z}$ let $g_{n}(x)=x^{3^{n}}$ and let $\mu_{n}: \mathscr{P}\left(g_{n}[V]\right) \rightarrow\left[0,2^{n}\right]$ be defined by $\mu_{n}\left(g_{n}[A]\right)=2^{n} \cdot \mu_{0}(A)$. Define 
$\mu: \mathscr{P}\left(\mathbf{R}^{1}\right) \rightarrow[0, \infty]$ by

$$
\mu(A)=\sum_{n \in \mathbf{Z}} \mu_{n}\left(g_{n}\left[A_{n}\right]\right)=\sum_{n \in \mathbf{Z}} 2^{n} \cdot \mu_{0}\left(A_{n}\right)
$$

where $A_{n} \subset V$ are such that $A \backslash\{0\}=\bigcup_{n \in \mathbf{Z}} g_{n}\left[A_{n}\right]$.

It is easy to see that $\mu$ is a $\sigma$-finite measure. Moreover,

$$
\mu\left(g_{m}[A]\right)=\mu\left(\bigcup_{n \in \mathbf{Z}}\left(g_{m} \circ g_{n}\right)\left[A_{n}\right]\right)=\sum_{n \in \mathbf{Z}} 2^{m+n} \cdot \mu_{0}\left(A_{n}\right)=2^{m} \cdot \mu(A),
$$

i.e., $\mu$ is $\gamma_{0}$-invariant where $\gamma_{0}: G_{0} \rightarrow(0, \infty)$ is defined by $\gamma_{0}\left(g_{n}\right)=2^{n}$. It is easy to see that $\gamma_{0}$ has little to do with a classical Jacobian.

Our group $G_{0}$ is countable. But if we consider a measure $\nu$ being a product measure of $\mu$ and a one-dimensional Lebesgue measure $m$ then $\nu$ is a $\sigma$-finite $\gamma$-invariant where $\gamma: G \rightarrow(0, \infty), G=\left\{\left(g_{n}, i\right): g_{n} \in G_{0}\right.$ and $i$ is an isometry of $\left.\mathbf{R}^{1}\right\}$, and $\gamma\left(g_{n}, i\right)=2^{n}$. It is also obvious that $G$ is uncountable. Moreover about $\nu$ we can prove that if $f$ is a homeomorphism of $\mathbf{R}^{2}$ and the system $\left\langle\mathbf{R}^{2}, \mu_{f}, G_{f}, \gamma_{f}\right\rangle$ is induced by $f$ from the system $\left\langle\mathbf{R}^{2}, \mu, G, \gamma\right\rangle$ then $G$ is not a subgroup of affine transformations of $\mathbf{R}^{2}$.

15. Problem 3. Is the assumption $H \subset\left(\mathbf{R}\left(X_{1}, \ldots, X_{n}\right)\right)^{n}$ essential in Theorem 3.1 ?

\section{REFERENCES}

[Be] A. Beardon, The geometry of discrete groups, Springer-Verlag, New York, 1983.

[CK] C. Chang and H. Keisler, Model theory, Studies in Logic and Foundations of Math., NorthHolland, 1977.

[Ci] K. Ciesielski, How good is Lebesgue measure?, Mathematical Intelligencer 11 (1989), 54-58.

[CP] K. Ciesielski and A. Pelc, Extensions of invariant measures on Euclidean spaces, Fund. Math. 125 (1985), 1-10.

[GR] R. C. Gunning and H. Rossi, Analytic functions of several complex variables, Prentice-Hall, Englewood Cliffs, N.J., 1965.

[Ha1] A. B. Harazisvili, On Sierpinski's problem concerning strict extendibility of an invariant measure, Soviet Math. Dokl. 18 (1977), 71-74.

[Ha2] _ Groups of transformations and absolutely negligible sets, Bull. Acad. Sci. Georgian SSR 115 (1984). (Russian)

[Hu] A. Hulanicki, Invariant extensions of the Lebesgue measure, Fund. Math. 51 (1962), 111-115.

[Je] T. Jech, Set theory, Academic Press, 1978.

[La] S. Lang, Algebra, Addison-Wesley, 1984.

[MW] J. Mycielski and S. Wagon, Large free groups of isometries and their geometrical uses, Enseign. Math. 30 (1984), 247-267.

[Pe] A. Pelc, Invariant measures and ideals on discrete groups, Dissertationes Math. 255 (1986).

[Pk] S. S. Pkhakadze, K teorii lehegovskoi miery, Trudy Tbiliss. Mat. Inst. 25 (1958). (Russian)

[Ro] A. Robinson, Complete theories, North-Holland, Amsterdam, 1956.

[Ru] W. Rudin, Rer'l and complex analysis, McGraw-Hill, 1987. 
[Sz] E. Szpilrajn, Sur l'extension de la mesure lebesguienne, Fund. Math. 25 (1935), 551-558. (French)

[We] B. Weglorz, Large invariant ideals on algebras, Algebra Universalis 13 (1981), 41-55.

Department of Mathematics, Warsaw University, Warsaw, Poland

Department of Mathematics, University of Louisville, Louisville, Kentucky 40292

Current address: Department of Mathematics, West Virginia University, Morgantown, West Virginia 26506 